\title{
Novel in -Situ heating transmission electron microscope holder for atomic resolution
}

\author{
Khaled Bataineh \\ Jordan University of Science and Technology, PO030, Irbid 2110, Jordan, E-mail: k.bataineh@just.edu.jo \\ cross $^{\text {ref }}$ http://dx.doi.org/10.5755/j01.mech.23.2.13544
}

\section{Nomenclature}

$c$ - specific heat, $\mathrm{J} / \mathrm{Kg}{ }^{\circ} \mathrm{C} ; \quad[D]$ - conductivity matrix, $\mathrm{J} / \mathrm{m}{ }^{\circ} \mathrm{C} ; F_{i j}$ - irradiation view factors; $F E A$ - finite element analysis; $\{q\}$ - heat flux vector, Watt; $\{\dddot{q}\}$ - heat generation rate per unit volume, Watt $/ \mathrm{m}^{3} ; Q$ - Total heat generation, Watt; $T$ - temperature, ${ }^{\circ} \mathrm{C}$; TEM - Transmission Electron Microscopy; $\{V\}$ - velocity vector, $\mathrm{m} / \mathrm{s} ; \rho$-density, $\mathrm{kg} / \mathrm{m}^{3} ; \sigma$-Stefan-Boltzmann constant; $\varepsilon$-effective emissivity of surface.

\section{Introduction}

Transmission Electron Microscopy is an imaging technique whereby a beam of electrons is focused onto a specimen causing an enlarged version to appear on a fluorescent or layer of photographic film, or to be detected by a CCD camera. The TEM is used heavily in the material and the biological sciences. For the TEM, it is essential to have the specimen thickness reduced to permit electron transmission. To achieve electron transparency, it is necessary to reduce the specimen thickness to less than 5000 Angstroms. Specimens are prepared by various, material dependent methods. They are typically a $3 \mathrm{~mm}$ diameter disk. For most electronic materials, a common sequence of specimen preparation techniques is ultrasonic disk cutting, dimpling and ion-milling [1].

In - situ TEM imaging means applying external conditions and influences (stimuli) that induce changes in the specimen and some method of recording and analyzing the resulting dynamic responses. For many years, a practical limitation has existed in TEM that precludes the ability to conduct in-situ experimentation. This is related to the physical gap between the microscope pole pieces. The pole pieces are individual components within the TEM's lens system that define the three dimensional space for the specimen. In a TEM, the specimen is placed into this pole piece gap. For ultra-high resolution TEMs, the pole piece gap is on the order of $2.2 \mathrm{~mm}$. It should be noted that smaller gaps yield higher resolution. Improvements in TEM technology have provided the ability to capture atomic scale; however, atomic scale stability is required for these measurements. These improvements have led to an increase in the sensitivity of the TEM to environmental disturbances. Environmental effects include, but not limited to, electromagnetic field, temperature changes, airflow across the column and air pressure changes. Currently, most TEM systems utilized CCD able to record images down to about several tens of milliseconds. Any drift, bending, or tilting of the specimen during recording images is a major limiting factor for high resolution dynamic ex- perimentations and can cause subnational measurement errors.

In-situ experimentations allow deeper understanding of important processes such as; hot deformation, dynamic recovery and dynamic recrystallization. These processes are not well understood because it is difficult to assess the nature microstructural processes solely from the traditional post mortem study of samples. Environmental in situ experiments are also important because many of the traditional techniques analyze catalyst samples under static, post-reaction conditions which are often not representative of the true dynamic state of a reacting catalyst. This has delayed a better fundamental understanding of dynamic catalytic surfaces, complex structural changes, and reaction mechanisms that evolve during oxidation-reduction processes. In contrast, in situ deformation experiments enable changes of microstructure and texture to be followed in a single region and enable information to be obtained about the mechanisms of microstructural evolution. In situ E-TEM experiments offer valuable insight into the chemical reaction processes.

Many reactions and transformations such as crystal growth, recrystallization, and phase transitions in solids take part at elevated temperatures. In situ TEM instrumentation allows discovery of completely new transformation phenomena in systems such as clusters of atoms, nanoparticles, nanotubes, or nanowires. Petkov presented a review of recent developments in in - situ TEM instrumentation and operation conditions [2]. The review demonstrated the improved fundamental understanding of physical phenomena associated with nanowire or nanotube materials due to in real time transformation at high resolution. In situ heating stages for TEM are usually attached to the specimen holder. A single tilt heating stage that can accommodate a $3 \mathrm{~mm}$ disk specimen and can reach $1200^{\circ} \mathrm{C}$ when using a Tantalum furnace is developed by Gatan Inc. [3]. High resolution TEM imaging at elevated temperatures requires high $\left(<0.1^{\circ} \mathrm{C} / \mathrm{min}\right)$ temperature stability and low sample drift. The large sample stage with very large thermal mass slows the temperature response which makes very difficult to obtain atomic resolution. To overcome this problem, heating stages have miniature stages were developed and are commercially available by Protochips Inc [4] and DENs solutions [5]. These utilized localised resistive heating elements to provide heat to the sample support. As a result of these commercially available heating holders, several studies conducted in-situ heating experiments under temperature ranges between 200 and $900^{\circ} \mathrm{C}$ [6-11]. Other investigators have built their own laboratory heating holder assemblies [12-13]. For example, Kamino et al. developed gas injection/specimen heating holder for the purpose of in situ observation of gas reaction of materials 
at high temperatures in a transmission electron microscope at near-atomic resolution. They used very fine tungsten wire as a heating element of the holder [12].

Zhang and Su studied the behavior of TEM metal grids during in-situ heating experiments [14]. They found that some grids at temperature between 650 and $850^{\circ} \mathrm{C}$ can generate nano-particles that can complicate experimental observations, particularly in in-situ heating studies of dynamic behaviors of nano-materials in TEM. Mortensen et al. modeled the temperature and velocity field, pressure distribution, and the temperature variation across the sample region inside an environmental transmission electron microscope (ETEM) by computational fluid dynamics (CFD) [15]. The simulation was made using the weakly compressible Navier - Stokes and general heat transfer packages of the commercial software program Comsol. They found that temperature drop from the furnace to the center of the sample grid was less than $10^{\circ} \mathrm{C}$ when simulating typical operating conditions. This finding verifies the isothermal conditions assumption (the temperature at the grid is very close to the furnace even in gaseous environments). However, their study is limited to furnace temperature up to $500^{\circ} \mathrm{C}$. From review of published papers, it is obvious that the designing of heating holder are based on observations and inaccurate measurements. Only few published papers presented formal analyses of the thermal and dynamic behavior of the holder.

The objective of this study is to develop heating holder that capable of elevating the sample temperature up to $2000 \mathrm{~K}$ while maintain atomic stability. Solid modeling and finite element simulations are used to assess the performance of the heating holder. The life of the holder and the stability of the sample are the main objective function. This study can be used as technical guidelines for design of optimal heating holder for accurate measurements. The indepth finite element results provide a deeper understanding of the thermal and dynamic behavior of the heating holder. It can provide engineering directions for design optimal heating holder for accurate measurements.

\section{Proposed design of heating holder}

The proposed design allows in-situ experimentations that is capable of elevating the temperature of a materials research specimen from ambient to $1770^{\circ} \mathrm{C}$ while simultaneously subjecting it to varying environments. The specimen temperature will be controlled to within $10^{\circ} \mathrm{C}$. Various environments will be created by injecting one or more gases into the volume surrounding the specimen. The specimen positioning within the TEM will be accurately controlled by the combination of the TEM's goniometer and the in-situ holder mechanism. The specimen will be positioned in 5 degree of freedom (DOF) space. These will include three translations $(\mathrm{X}, \mathrm{Y}, \mathrm{Z})$ and two tilt axes $(\alpha, \beta)$ with a targeted accuracy of $+/-0.005^{\circ}$.

The proposed design incorporates resistive heating technology as shown in Fig. 1. Heating elements have been encased in a ceramic insulator to prevent electrical shorts in the heating coils. The supporting mechanisms for the heating elements are designed to minimize the contact area (thus conductive heat transfer). The proposed technology also includes a radiation shield surrounding the heater assembly to protect the TEM pole pieces. In the proposed model, the specimen loading and retention mechanisms are not shown for clarity. Design restrictions on the dimensions of the holder tip as a function of a $\sim 6 \mathrm{~mm}$ pole piece gap are satisfied. Since this proposed design will ultimately be compatible with different microscope stages, generic dimensions were applied to the holder barrel. Tungsten was chosen for the heater coils, the heat shield, sample rings, and the enclosure that covers the electrical insulator. Four $1 \mathrm{~mm}$ diameter spheres made of $\mathrm{Al} 2 \mathrm{O} 3$ provide symmetrical support for the specimen containing the furnace assembly. Those spheres are attached to a spring like outer support structure to allow expansion without inducing significant stresses. $34 \mathrm{AWG}$ wire was chosen for the heater coils. This geometry will allow $26.4 \mathrm{~cm}$ of tungsten wires to be used for the heater.

Increasing the resistance of the coils is one of the design parameters. This will allow large wattage with minimum amperage, and hence increase the heaters life. The contact area between the spheres and outer shell need to be minimized to increase the thermal resistance and keep the heat in a very localized area. Our current proposed design has a contact area of $9 \times 10^{-8} \mathrm{~m}^{2}$ at four locations. The function of the shield is to maintain the heat inside the sample chamber. Since the reflected radiation does not change the internal energy of the reflective material, using high reflective material will have the advantages of minimizing the heat loss by increasing the resistance of heat flux out of the furnace. This will also serve to protect of the microscope components and reduces the generated nano- particles that affect the measurements.

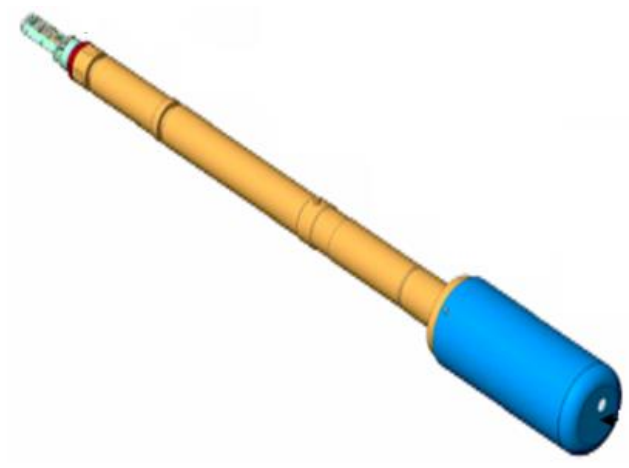

a

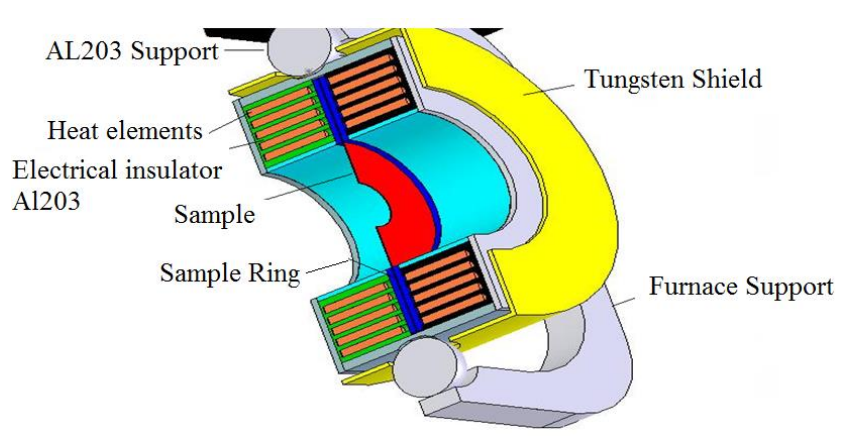

b

Fig. 1 Solid Model for the proposed heating holder [16]. $\mathrm{a}$ - Heating holder; $\mathrm{b}$ - zoom at the tip of the heating holder

\section{Finite element analyses}

Heat distribution in space and time with the heating holder is considered highly complex problem involves 
several complex mechanisms. It is not possible to theoretical determine the evolution, in space and time, of the temperature distribution with the heating holder. In order to overcomes this limitation, finite element analyses were carried out to quantify the performance of the design using commercially available software ANSYS [17]. This type of analysis will be used to qualify the components and assemblies in terms of mechanical stress, thermal loading dynamic mechanical behavior.

\subsection{Heat transfer fundamentals}

This section presents an overview of the governing equations incorporated by ANSYS for thermal analysis. The first law of thermodynamics states that thermal energy is conserved. The differential equation governing the heat flow on a differential volume is:

$$
\rho c\left(\frac{\partial T}{\partial t}+\{V\}^{T}[L] T\right)+[L]^{T}\{q\}=\dddot{q}
$$

where $\rho$ is density; $c$ is specific heat; $T$ is temperature $T(x, y, z, t) ;[L]$ is a vector operator; $[L]=\left\{\frac{\partial}{\partial x}, \frac{\partial}{\partial y}, \frac{\partial}{\partial z}\right\}$, $\{V\}$ is the velocity vector for mass transport of heat, $\{q\}$ is heat flux vector, $\{\dddot{q}\}$ is the heat generation rate per unit volume, Fourier's law is used to relate the heat flux vector to the thermal gradients:

$$
\{q\}=-[D]\{L\} T,
$$

where $[D]$ is conductivity matrix given as:

$$
[D]=\left[\begin{array}{ccc}
K_{x x} & 0 & 0 \\
0 & K_{y y} & 0 \\
0 & 0 & K_{z z}
\end{array}\right]
$$

There are three types of boundary conditions encountered in solving thermal problems: specified temperatures acting over a surface, specified heat flows acting over a surface and specified convection acting over surface (Newton's law of cooling).

Radiation is the transfer of energy via electromagnetic waves. The waves travel at the speed of light, and energy transfer requires no medium. Thermal radiation is just a small band on the electromagnetic spectrum (i.e. wavelength $\lambda$ ranges between 0.1 and $100 \mu \mathrm{m}$ ). Because the heat flow that radiation causes varies with the fourth power of the body's absolute temperature, radiation analyses are highly nonlinear. ANSYS introduces radiation effects only through the boundary conditions; the coupling is especially strong due to nonlinear dependence of radiation on surface temperature. The heat transfer rate between surfaces $i$ and $j$ radiating to each other is:

$$
Q_{i}=\sigma \varepsilon_{j} F_{i j} A_{j}\left(T_{i}^{4}-T_{j}^{4}\right)
$$

where $T_{i}, T_{j}$ are the absolute temperature at surface $i$ and $j$, respectively, $Q_{i}$ is energy loss of surface $i, \sigma$ is StefanBoltzmann constant, $\varepsilon_{i}$ is effective emissivity of surface $i$, $F_{i j}$ is radiation view factors, $A_{i}=$ area of surface $i$. The view factor, $F_{i j}$, is defined as the fraction of total radiant energy that leaves surface $i$ which arrives directly on surface $j$. Emissivity is a surface radiative property defined as the ratio of the radiation emitted by the surface to the radiation emitted by a black body at the same temperature. ANSYS restricts radiation exchange between surfaces to gray-diffuse suraces. The word grey signifies that the emissivity and absorptivity of the surface do not depend on wavelength. The word diffuse signifies that emissivity and absorptivity do not depend on direction. For a gray diffuse surface, emissivity=absorptivity; emissivity + reflectivity $=1$. A black body surface has a unit emissivity.

\subsection{Finite element model and boundary conditions}

The solid models are directly transferred into ANSYS software for the execution of Finite Element Analysis (FEA). Thermal-stress analysis is used to take into account the interaction (coupling) between thermal and stresses due to thermal expansion or contraction. Heat transfer analysis was used to obtain the temperature distribution of model, the heat power requirements to obtain the desired temperature, the temperature distribution on the sample, and finally the temperature varying with time especially at the goniometer stage. After obtaining thermal results, structural analyses were carried out to obtain the thermal stresses and displacements of the model parts.

The actual Solid Model shown in Fig. 1 was imported into ANSYS. The material properties which were input to the finite element model are listed in Table 1. The physical parameters for tip area are assumed independent of temperature [15]. The model consisted of about 200,000 elements. All solid elements were of type SOLID87 and SOLID90. SOLID90 is a 3-D thermal element that has 20 nodes with a single degree of freedom, temperature, at each node. The 20-node elements have compatible temperature shapes and are well suited to model curved boundaries. SOLID87 is a 3-D 10 node element that is well suited to model irregular meshes (such as produced from various $\mathrm{CAD} / \mathrm{CAM}$ systems). The element has one degree of freedom (temperature) at each node. These elements are applicable to a 3-D, steady-state or transient thermal analysis.

Three modes of heat transfer (conduction, convection and radiation) were included in all simulations. The Radiosity Solver Method was used to solve the radiation thermal analysis. This method works for generalized radiation problems involving two or more surfaces receiving and emitting radiation. The method is supported by all 3D/2-D elements having a temperature degree of freedom. The heating elements were modelled as solid cylinders with diameters comparable the 34AWG. The total length of those cylinders was set equal to the total length of the resistors. Heat generated was applied as a body force on the heating elements. At the inlet of TEM holder, room temperature $\left(25^{\circ} \mathrm{C}\right)$ is specified as boundary condition. Electron beam effects were completely ignored in the simulations.

The conduction of heat through homogenous, isotropic materials has been well developed; however, the heat conduction across interfaces formed by contacting surfaces is still under investigations. In order to accurately determine the heat distribution within the specimen, it is necessary to understand the heat flow behaviour through 
contact surfaces. The actual contact area between two surfaces is less than the apparent area of contact. The amount of actual contact area depends on physical properties of the contacting materials, surface roughness, surface waviness, contacting pressure. It should be recalled that uniform distribution of temperature within specimen is one of the vital requirements for heating holder. Deshmukh investigated the effect of thermal contact conductance on the temperature distribution with the specimen [18]. It was found that if clamping pressure is sufficient, the value of thermal contact conductance ranges between $0.001-0.00001{ }^{\circ} \mathrm{C} / \mathrm{Wm}^{2}$ can be assumed. In this study, all thermal contact resistances were set to constant values $0.0001^{\circ} \mathrm{C} / \mathrm{Wm}^{2}$.

Heat transfer by convection is considered highly complicated problems. It depends on the fluid velocity, fluid viscosity, flow rate, heat flux, and type of the flow. In order to analyse the effect of convection on the specimen temperature, several values of heat convection coefficient are studied. Convection heat transfer was applied as a surface load on the part of the model that is not under vacuum condition. Fig. 2 shows the boundary conditions that have been applied for the entire simulation. Since the TEM is placed inside a clean quite room, it is expected that the free convection coefficients takes small values ranging between $10-20 \mathrm{~W} / \mathrm{m}^{2}{ }^{\circ} \mathrm{C}$. Fig. 3 shows the effect of convection coefficient on the sample temperature. As can be seen in Fig. 3, the specimen temperature does not vary significantly with heat convection coefficients range studied. We chose a value of $15\left(\mathrm{~W} / \mathrm{m}^{2}{ }^{\circ} \mathrm{C}\right)$ for the heat convection coefficient at the very end of the holder. A lower value was chosen on the part of the holder that is inside the microscope up to the vacuum seal. Under vacuum conditions there are only two modes of heat transfer (radiation and conduction). Because the heat flow that radiation causes varies with the fourth power of the body's absolute temperature, radiation analyses are highly nonlinear and become more dominant at high temperatures. For this reason, we chose to ignore the heat radiation (heat losses) in the part of the models that are known to operate under relatively low temperatures $\left(<100^{\circ} \mathrm{C}\right)$.

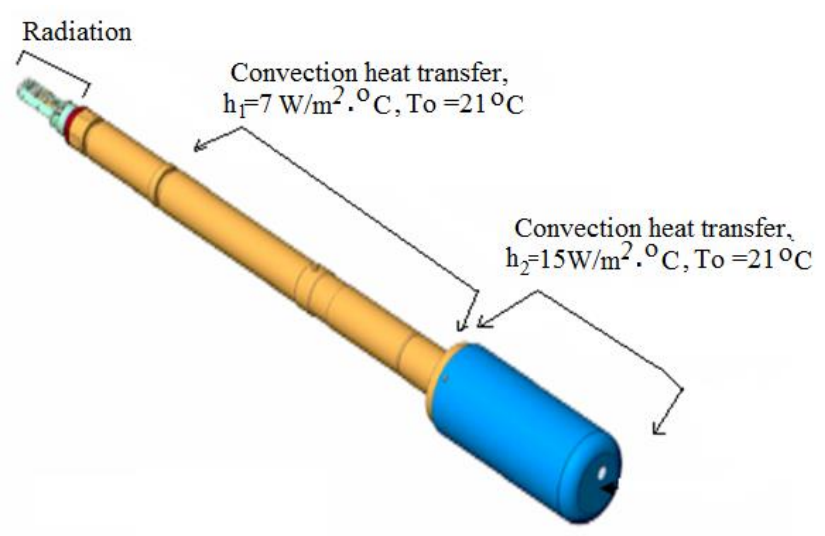

Fig. 2 Boundary conditions

Radiation heat transfer was applied between all tip surfaces. The emissivities of the tip material were set constant with temperature. ANSYS assumes heat transfer by radiation as a surface property and not volumetric. That assumption is valid since in most solids, radiating from interior molecules is strongly absorbed by adjoining molecules. The objects surrounding the furnace are treated as radiative surfaces. Since the tip area has an open enclosure, ANSYS requires specification of a space temperature for energy balance to the ambient. We chose a space temperature to be $50^{\circ} \mathrm{C}$. The heat generating due to current passing through resistance is modelled as a body force on the heater coils. The value of this body force is computed by dividing the input power by the total volume of the heater coils. The total volume of the heaters is $6.4 \times 10^{-9} \mathrm{~m}^{3}$, and heat generation is $Q=3.0 \times 10^{9} \mathrm{~W} / \mathrm{m}^{3}$.

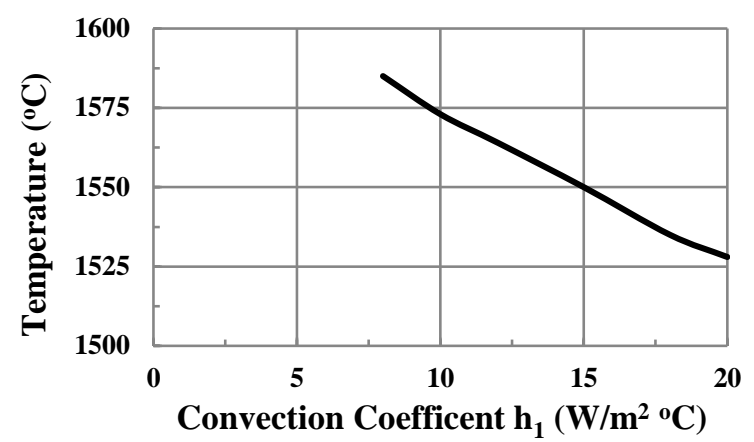

Fig. 3 Variation of maximum specimen temperature with convection coefficient

Table 1

Material Properties

\begin{tabular}{c|c|cc}
\hline \hline Material & $\begin{array}{c}\text { Density, } \\
\mathrm{kg} / \mathrm{m}^{3}\end{array}$ & $\begin{array}{c}\text { Thermal Conduc- } \\
\text { tivity, W/m }{ }^{\circ} \mathrm{C}\end{array}$ & $\begin{array}{c}\text { Modulus of } \\
\text { elasticity, } \mathrm{Pa}\end{array}$ \\
\hline Tungsten & 19,300 & $\begin{array}{c}163.0 \text { at } 25^{\circ} \mathrm{C} \\
100 \text { at } 1800^{\circ} \mathrm{C}\end{array}$ & $4.0 \times 10^{11}$ \\
& & $\begin{array}{c}30 \text { at } 25^{\circ} \mathrm{C} \\
5 \text { at } 1800^{\circ} \mathrm{C}\end{array}$ & $3.7 \times 10^{11}$ \\
$\mathrm{Al} 2 \mathrm{O} 3$ & 3960 & 60.5 & $2.0 \times 10^{11}$ \\
Copper & 8300 & & \\
\hline \hline
\end{tabular}

\section{Results and discussion}

\subsection{Existing heating holder technology}

Fig. 4 shows a schematic conceptual solid model of the tip heating holder technology available commercially. The commercial heaters utilized several heater coils attached to ceramic plate and shielded by tungsten shield. A comprehensive simulation model using FEM for the analysis of transient heat transfer of the holder assembly is built. Simulation is used to predict the temperature distribution in the holder and sample. The main objectives of these investigations are to gain experience with the current holder assemblies and identify their limitations and drawbacks.

Fig. 5 shows the coils and sample temperature variation with time obtained from transient analysis. 19.2 Watts was applied to the heater coils as heat generation. The steady state temperature of the coils is $1500^{\circ} \mathrm{C}$ and the sample temperature is $1010^{\circ} \mathrm{C}$. The difference between the temperature of the sample and the heater coils is on the order of $500^{\circ} \mathrm{C}$. This large difference is the reason for short life of the heater coils when this technology is used at elevated temperature. The heater coils have to operate at much higher temperatures (on the order of $1900^{\circ} \mathrm{C}$ ) to ele- 
vate the sample temperature to $1200^{\circ} \mathrm{C}$. Design optimization was carried out to reduce temperature difference between the heater coils and the sample. Since the radiation is the dominant heat transfer mode, focus is on optimizing radiation factors. Radiation depends on: the temperature difference between the load and the source, viewing factor, emissivity of radiating surfaces, and surface area of radiating surfaces. Viewing factor can be increased by reducing the distance between the sample and the heater coils (viewing factor is inversely proportional to the square of the distance). Further optimization could be achieved if the emissivity of the heater coils is increased by applying a special coating. Fig. 6 shows the temperature distribution of the sample after it has reached steady state. It can be seen in Fig. 6 that the distribution is not uniform. The parts that were very close to the heater coils reach a higher temperature compared to the rest. At locations where the specimen is very thin for electron transparency (close to the hole), the simulation predicted significant temperature gradient.

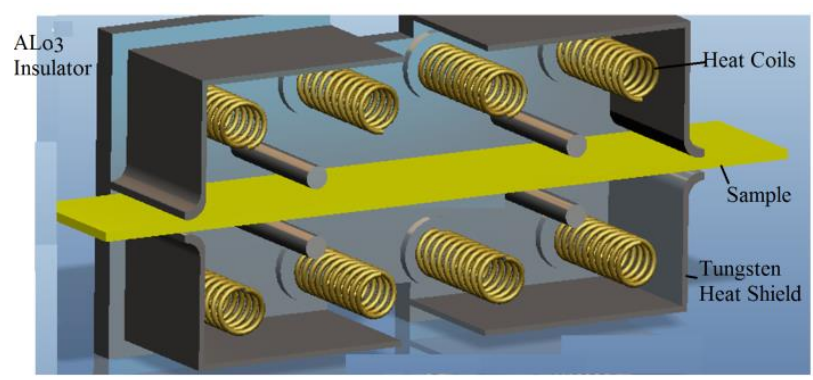

Fig. 4 Conceptual design of the tip of commercial Heating Holder technology

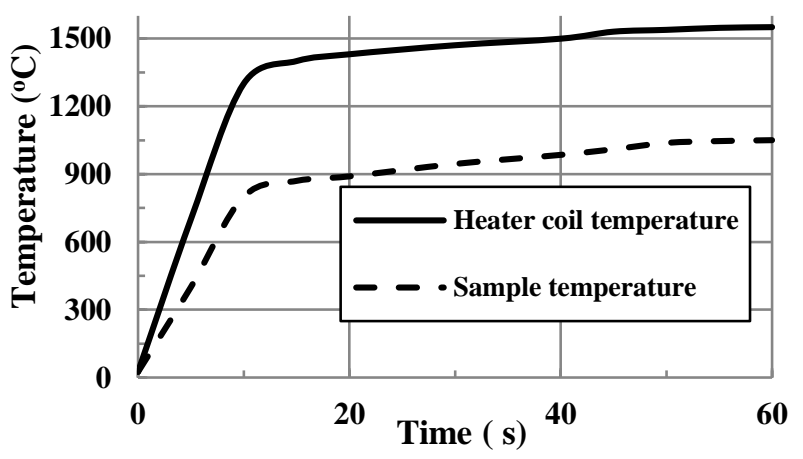

Fig. 5 Transient coils and sample temperature $\left({ }^{\circ} \mathrm{C}\right)$

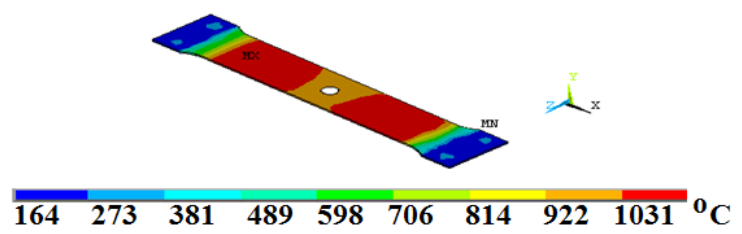

Fig. 6 Steady state temperature distribution of the sample [16]

\subsection{Proposed technology performance}

Based on the thermal behavior of the existing technology, the new design is proposed as shown in Fig. 1. In the FEA simulations, a power level of 9.6 Watts was applied to the heating elements. Fig. 5 shows the transient temperature of the heater, sample, spheres, and at location of $1 \mathrm{~cm}$ from the tip area. The heater's temperature reached a steady state of value $1770^{\circ} \mathrm{C}$ after 70 seconds. The sample temperature was $40^{\circ} \mathrm{C}$ lower than the heater. This is one of the advantages of the proposed heaters compared to the existing heaters. It is interesting to note that at a location $1 \mathrm{~cm}$ from the tip, the temperature was still increasing. New simulations need to run for longer time periods to obtain the variation in temperature with time. It is very important to keep the temperature near the stage area within $\pm 1^{\circ} \mathrm{C}$. This is due to the fact that many investigators reported distortions in image data sets due to movements of the microscope stages that resulted in fluctuation in room temperature. The same analogy can be used here. Fig. 8 shows the temperature variations with time at a position $5 \mathrm{~cm}$ removed from the specimen area. This location was investigated since it is the position where the microscope goniometer mates with the holder. Note that the temperature at that position reached steady state after 80 minutes. The most important thing is there are no variations after 80 minutes. Fig. 9 shows the transient sample radial deformation as the sample is heated to the desired temperature. It is easy to see from Fig. 9 that the sample experiences continuous deformation while the sample is being heated. This amount of deformation can be easily reduced with modifying the design to allow higher values of friction coefficient between the sample and the sample ring. However, as mentioned previously, design this technology with higher friction will increase the amount of stresses in the sample. A successful design will utilize a compensation mechanism to account for the sample movements.

Fig. 10 shows the thermally-induced radial stresses developed in the specimen. The sample rings provide frictional support to the sample. The frictional coefficient of 0.4 was used. The magnitude is on the order of $3 \mathrm{MPa}$. Those stresses arise due to friction between the sample and the sample ring. As a design consideration, it will be necessary to capture the specimen in such a manner that takes into consideration this amount of stresses. Rigidly mounting the sample will decrease the amount of expansion; however, it will produce significant stress. The corresponding friction between the specimen and capturing mechanism will be investigated and optimized. Fig. 11 and Fig. 12 show the stress contours of the bracket that support the furnace. The maximum stresses at the support of the sphere due to localized stress concentrations.

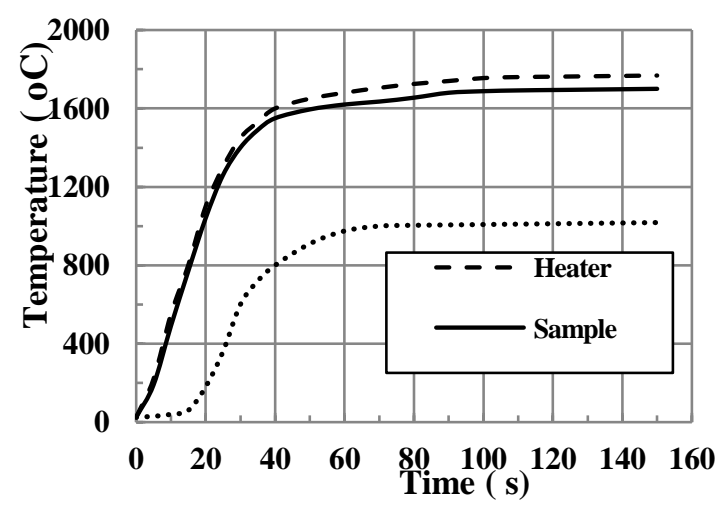

Fig. 7 Transient temperature $\left({ }^{\circ} \mathrm{C}\right)$ 


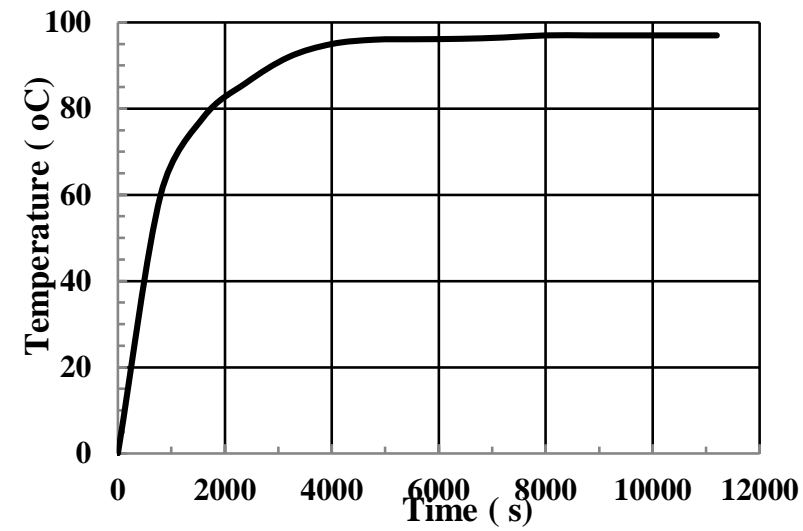

Fig. 8 Transient temperature at the $5 \mathrm{~cm}$ removed from the tip area

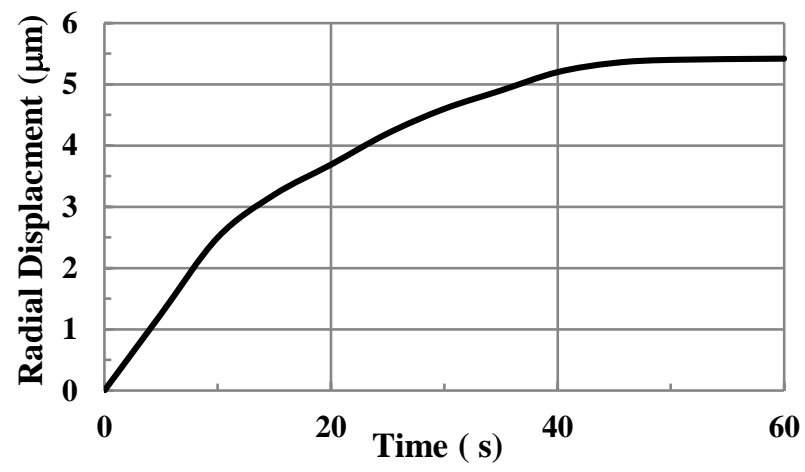

Fig. 9 Sample radial deformation during transient heating
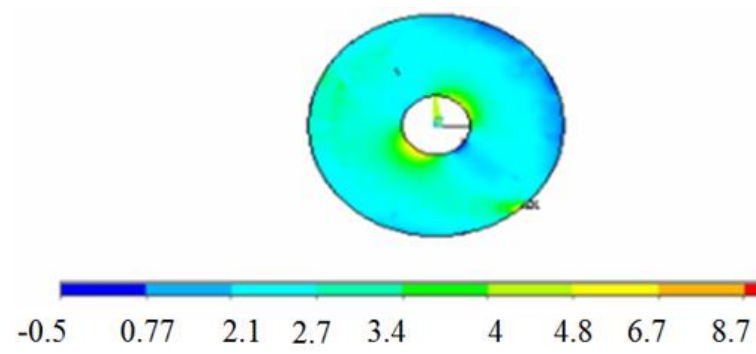

Fig. 10 Radial stresses $(\mathrm{Pa})$

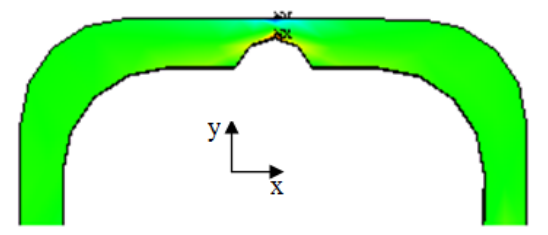

$\begin{array}{llllllllll}-16 & -13 & -9.4 & -5.7 & 2.3 & 3.4 & 5.3 & 8.7 & 32 & \mathrm{MPa}\end{array}$

Fig. $11 \mathrm{Y}$-direction stresses $(\mathrm{MPa})$ in the bracket
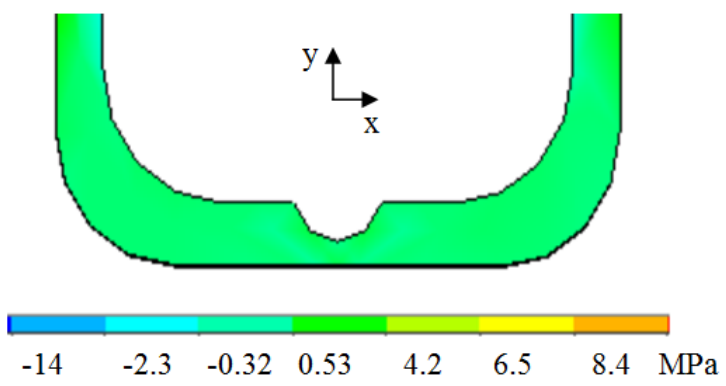

Fig. $12 \mathrm{X}$-direction stresses $(\mathrm{MPa})$ in the bracket

\section{Laser heating (Tomography)}

As an alternative design, this section investigates utilizing a localized laser diode for heating a specimen to $2000 \mathrm{~K}$. This technology has not been considered previously in TEM applications. Since resistive heating requires space to insert the resistors into the holder tip, the overall depth will restrict the possibilities of using this technology for tomography. A $\mathrm{CO} 2$ laser is distinguished by its much higher efficiency and extremely high power output. The $\mathrm{CO} 2$ laser wavelength is $10.6 \mu \mathrm{m}$ and falls in the range of infrared radiation, which is also referred to as thermal radiation. The interaction of this radiation with matter is thus thermal. The coherence of the laser beam permits it to be focused on a diameter of $10 \mu \mathrm{m}$. This design style will include a laser source which is separate from the holder assembly. The laser will be transmitted to the specimen cup via a fiber optic bundle. This fiber optic may be mounted through the body of the holder or through an alternate port.

Fig. 13 shows a solid model of the proposed design. The overall depth of the specimen cup is $1.5 \mathrm{~mm}$, which allows the tip to be tilted up to \pm 70 degrees. The irradiating heat from a $\mathrm{CO} 2$ laser was modeled as heat generation on a very small element on the specimen cup. Transient heat transfer analyses were carried out, similar to the above simulations. The irradiating heat from the $\mathrm{CO} 2$ laser was modeled as a heat generation on a very small element of volume on the order of $1 \times 10^{-13} \mathrm{~m}^{3}$.

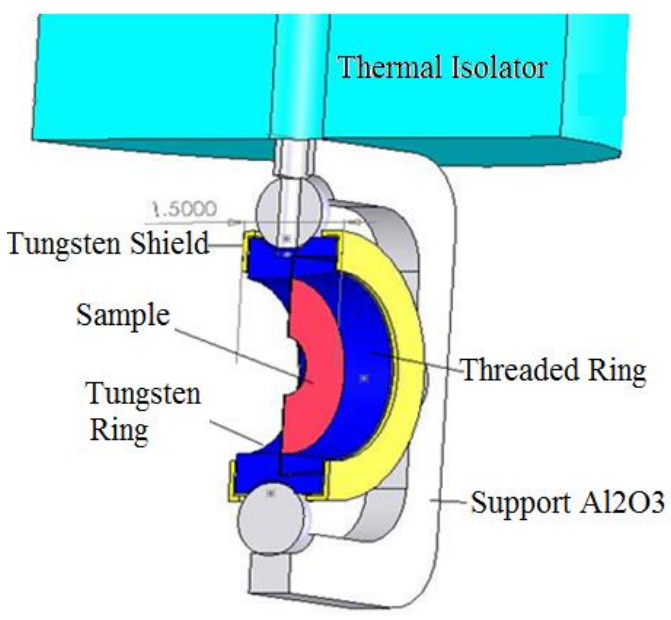

Fig. 13 Tomography holder utilizing laser heating

Simulations were carried out to predict the temperature distribution of the holder due to localized intensive heat generation. Simulations were carried out assuming that the $\mathrm{CO} 2$ laser is striking only one area. Results obtained will be used to check the design performance. The uniformity of the temperature distribution among the sample and the highest temperature of the target will be used as design criteria. A 4.6 Watts were supplied to an element of volume $\sim 4.8 \times 10^{-13} \mathrm{~m}^{3}$. Fig. 14 shows the temperature contour of the sample. The difference in temperature is on order of $80^{\circ} \mathrm{C}$. A successful design will reduce the temperature gradient within the sample.

For comparison purposes, our current finite element simulations are compared against those presented by Deshmukh [18]. Deshmukh presented temperature distribution for specimen cup for heating holder assembly using 
laser heat technology. They applied 4 Watt as surface heat flux to their holder assembly. Their results showed that the maximum cup temperature was $1698.4^{\circ} \mathrm{C}$ reached within 60 seconds reaches. Furthermore, their finite element simulation predicts that the temperature gradient across specimen cup was $580^{\circ} \mathrm{C}$. Since the present study applied more heat, the sample temperature is greater than those reported by [18]. Our finite element simulations predicted lower values of temperature gradient compared to those reported by [18]. The reason for this mismatch is due to the values of thermal conductance contact; present value of thermal conductance contact is 10000 while the Deshmukh used 1000 .

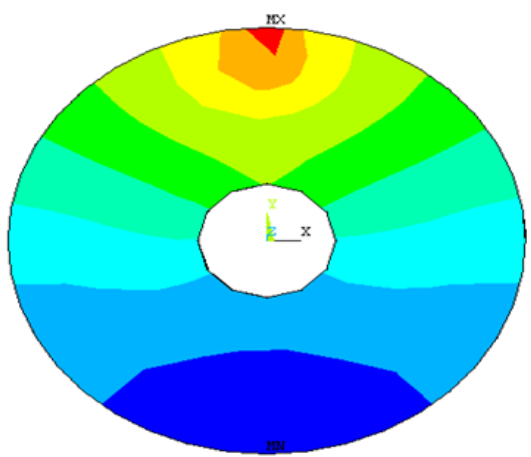

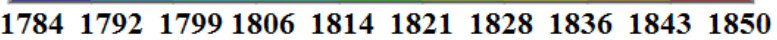

Fig. 14 Temperature contour of the sample $\left({ }^{\circ} \mathrm{C}\right)$
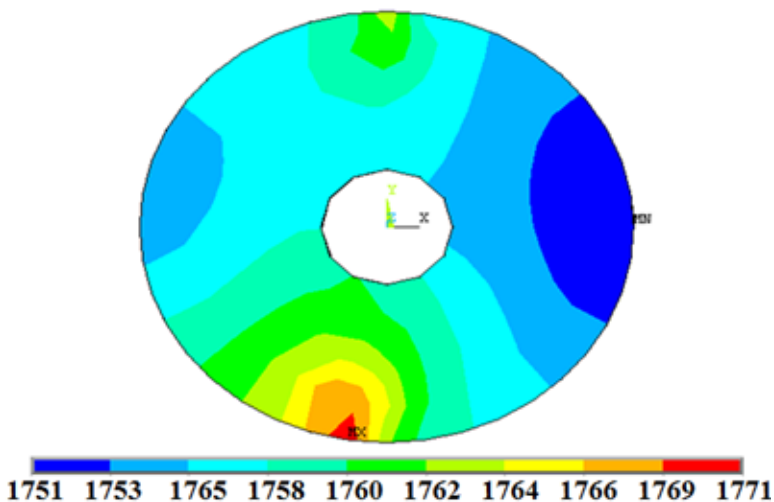

Fig. 15 Temperature contour for the sample $\left({ }^{\circ} \mathrm{C}\right)$

To obtain a more uniform temperature distribution, we modified the design so that the laser beam strikes two opposite areas. Fig. 15 shows the temperature contours for the ring. Using the modified design results in a more uniform distribution compared to the original design. For example, in the area where is the sample is very thin, the temperature can range between 1753 and $1757^{\circ} \mathrm{C}$ with the double target area design. The locations where the holder mates the goniometer stages are very critical for the holder stability and hence image stability. Any expansion due to temperature increase in that part will lead to undesirable movements of the holder and hence the sample. To restrict this undesirable temperature increase, this design uses a large mass of thermal resistance material between the holder tip and the rest of the holder body. Also this design utilize a very small area of contact between the bracket support and the sample ring to further increase the thermal resistance and hence reduce the heat flux to the rest of the holder body. This has advantages over the current com- mercial holders that use cooling water to maintain the holder rod temperature close to the stage temperature. We believe that using circulating cooling water will induce vibration and hence reduce the stability and the resolution that can be obtained.

\section{Conclusions}

Transient heat transfer analyses using FEA were carried out for novel resistive heating holder. Results were obtained for temperature distributions, thermal stresses and sample drift. The mass of the specimen furnace in both resistive and laser heating has been made as small as possible to ensure rapid response to changes in heater current and minimum wattage usage. The rate of increase of specimen temperature can be controlled as required, to a maximum of $3600^{\circ} \mathrm{C}$ per minute, or held at any selected temperature for long periods for the study of diffusion processes and precipitation kinetics. This technology has been designed to have minimum thermal drift. To achieve minimum thermal drift, heat loss from the furnace to the specimen tip has been minimized by careful design of the ceramic furnace supports. Such careful design results in the specimen rod temperature held as closely as possible to the temperature of the specimen stage. Based on finite element results, the proposed design outperforms the current technology in several aspects i.e more uniform temperature distributions within the sample, sample temperature close the heater temperature, symmetrical expansion of the sample, minimum thermal induced stresses in the sample, minimum heat losses to the TEM stages, lower generated nano particles from the TEM grids. To increase the stability due to temperature variations, a material with a near zero coefficient of expansion should be used for the holder part that mates the goniometer stage.

\section{References}

1. Kelly, F.; Holzma, D.; Elli, D. 1992. Mater. Res. Soc. Proc. 254, 79-96.

2. Petkov, N. 2013. In situ real-time TEM reveals growth, transformation and function in one-dimensional nanoscale materials: from a nanotechnology perspective, ISRN Nanotechnology, $21 \mathrm{p}$. http://dx.doi.org/10.1155/2013/893060.

3. http://www.gatan.com/products - checked on July 20, 2015.

4. http://www.protochips.com/products/aduro.htmlchecked on July 21, 2015.

5. http://www.denssolutions.com/en/our-products/sampleheating-systems - checked on July 22, 2015.

6. Romankov, S.; Park, Y.C. 2015. TEM study of microstructural development during heating in a nanolaminated amorphous $\mathrm{ZrAlCuFe} /$ crystalline $\mathrm{CuCoFeNi}$ composite structure, Journal of Alloys and Compounds 619: 417-429. http://dx.doi.org/10.1016/j.jallcom.2014.08.256.

7. Romankov, S.; Park, Y.C. 2015. In situ hightemperature TEM observation of material escape from a surface of $\mathrm{CoFeNi} / \mathrm{Cu} / \mathrm{ZrAlO}$ composite into the amorphous carbon layer, Journal of Alloys and Compounds 632: 408-416.

http://dx.doi.org/10.1016/j.jallcom.2015.01.214. 
8. Akita, T.; Tanaka, K.; Kohyama, M.; Haruta, M. 2007. Analytical TEM study on structural changes of $\mathrm{Au}$ particles on cerium oxide using a heating holder, Catalysis Today 122(3-4): 233-238. http://dx.doi.org/10.1016/j.cattod.2007.01.014.

9. Rashkova, B.; Faller, M.; Pippan, R.; Dehma, G. 2014. Growth mechanism of $\mathrm{Al} 2 \mathrm{Cu}$ precipitates during in situ TEM heating of a HPT deformed $\mathrm{Al}-3 \mathrm{wt} . \% \mathrm{Cu}$ alloy, Journal of Alloys and Compounds 600(5): 43-50. http://dx.doi.org/10.1016/j.jallcom.2014.02.090.

10. Yoo, S.J.; Kim, C.Y.; Shin, J.W.; Lee, S.G.; Jeong, J.M.; Kim, Y.J.; Lee, S.H.; Kim, J.G. 2013. Characterization of an amorphous carbon film covering a Mo grid during in situ heating TEM study, Materials Characterization 78 : 31-36.

http://dx.doi.org/10.1016/j.matchar.2013.01.011.

11. Sachin, L.; Shrestha, K.; Xie, Y.; Ringer, S.P.; Carpenter, K. R.; Smith, D.R.; Killmore, C.R.; Cairney J.M. 2013. The effect of clustering on the mobility of dislocations during aging in Nb-microalloyed strip cast steels: In situ heating TEM observations, Scripta Materialia 69(6): 481-484. http://dx.doi.org/10.1016/j.scriptamat.2013.06.007.

12. Kamino, T. et al. 2005. Development of a gas injection/specimen heating holder for use with transmission electron microscope, J Electron Microsc (Tokyo) 54(6): 497-503.

http://dx.doi.org/10.1093/jmicro/dfi071.

13. Yaguchi et al. 2010. Development of an Environmental TEM and specimen holders for a wide range of atmospheres and temperatures. AMTC Letters, vol. 2: 84-85.

14. Zhang, Z.; Su, D. 2009. Behaviour of TEM metal grids during in-situ heating experiments, Ultramicroscopy 109(6): 766-774.

http://dx.doi.org/10.1016/j.ultramic.2009.01.015.

15._Mortensen, P.M.; Hansen, T.W.; Wagner, J.B.; Jensen, A.D. 2015. Modeling of temperature profiles in an environmental transmission electron microscope using computational fluid dynamics, Ultramicroscopy 152: $1-9$. http://dx.doi.org/10.1016/j.ultramic.2014.12.007.

16. Bataineh, K. 2005. Development of precision tem holder assemblies for use in extreme environments, Doctoral Dissertation, University of Pittsburgh.
17. ANSYS Release 10.0, Multiphysiscs, Documentation Preview, Canonsburg, PA, USA: ANSYS Inc., 2005.

18. Deshmukh, P. 2008, Design and development of an environmental cell for dynamic in situ observation of gas- solid reaction at elevated temperature, PD thesis, University of Pittsburgh, Pittsburgh, USA.

\section{Khaled Bataineh}

\section{NOVEL IN - SITU HEATING TTRANSMISSION ELECTRON MICROSCIOPE HOLDER FOR ATOMIC RESOLUTION}

\section{S u m m a r y}

This study presents novel design of "in-situ" TEM heating holder. In-situ experimentations are important because hot deformation, dynamic recovery and dynamic recrystallization are important processes which are not well understood because it is very difficult to assess the nature microstructural processes solely from the traditional post mortem study of samples. We have designed an optimum heating holder that is dynamically stable for accuracy and image processes, capable of elevating the sample temperature up to $2000 \mathrm{~K}$, with minimum controllable drift of the sample position. Sample drift is calculated. Thermal stressed induces in the sample are calculated. The in-depth finite element simulations provide detailed information about thermal and dynamic behavior of the heating holder. Finite element results show that the proposed design outperform the current heating technology. Based on the finite element results, a revised design is proposed that has lower temperature gradient among the sample, lower temperature difference between the sample and the TEM grids, lower thermal stresses in the sample, lower heat losses to the microscope parts. Furthermore, the present analysis provides the basis for the design of optimal heating holder for accurate measurements.

Keywords: In- situ TEM instrumentation, Heating holder, finite element analysis, atomic resolution.

Received October 30, 2015

Accepted April 14, 2017 